\title{
Study of Smart Grid Communication Network Architectures and Technologies
}

\author{
Naeem Raza1, Muhammad Qasim Akbar ${ }^{2}$, Aized Amin Soofi ${ }^{3}$, Samia Akbar ${ }^{2}$ \\ ${ }^{1}$ Department of Computer Science, National Textile University, Faisalabad, Pakistan \\ ${ }^{2}$ Department of Computer Science, Government College University, Faisalabad, Pakistan \\ ${ }^{3}$ Department of Computer Science, Allama Iqbal Open University, Islamabad, Pakistan \\ Email: naeemraza1248@gmail.com,emqa1408@gmail.com, aizedamin@yahoo.com, samiaakbar45@gmail.com
}

How to cite this paper: Raza, N., Akbar, M.Q., Soofi, A.A. and Akbar, S. (2019) Study of Smart Grid Communication Network Architectures and Technologies. Journal of Computer and Communications, 7, 19-29.

https://doi.org/10.4236/jcc.2019.73003

Received: January 9, 2019

Accepted: March 12, 2019

Published: March 15, 2019

Copyright (c) 2019 by author(s) and Scientific Research Publishing Inc. This work is licensed under the Creative Commons Attribution International License (CC BY 4.0).

http://creativecommons.org/licenses/by/4.0/

Open Access

\begin{abstract}
Smart Grid (SG) is an emerging paradigm of the modern world to upgrade and enhance the existing conventional electrical power infrastructure from generation to distribution to the consumers in a two-way communication fashion to automate the electrical power demand and supply and make this a cyber-physical system. SG infrastructure key elements, such as smart meters, circuit breakers, transformers, feeders, substations, control centers, grid stations, are required well-formed communication network architectures. SG infrastructure is divided into three main communication networks architectures, such as HAH, NAN, and WAN. Each of these communication network architectures requires reliable, stable, secure, high data rate at real-time with the help of different wireline and wireless communication technologies from HAN to WAN networks. To understand the complete concepts about SG, a concise review is presented and it will help the readers to get foundations of communication network architectures and technologies of SG.
\end{abstract}

\section{Keywords}

Smart Grid, Communication Network Architectures, Wireline and Wireless Communication Technologies, HAN, NAN, WAN

\section{Introduction}

This Electricity demand of any country is increasing day by day and causes the emergence of several severe issues regarding congestion, safety, lack of ubiquitous and operational communication, fault diagnoses, monitoring and automation due to the nonlinear and complex distribution of electrical power. These problems may cause a major breakdown at a regional level or beyond by just 
having a cascading effect on a minor fault. Consequently, it's a global concern of $21^{\text {st }}$ century to have a different alternative and renewable energy source to take demand of power by addressing several new design challenges such as storage of energy, the stability of the power systems and integration of power grids [1]. Smart Grid will enhance the capabilities of the traditional energy systems and make available for us a more advanced and automated future energy system. They have lots of characteristics, such as distributed control, solar or wind-based energy productions, novel components, and virtual smart power plants etc. [2]. SGs are power systems based on integrated bidirectional communications by sensing and control through the different technologies [3]. Modern SGs are capable of providing effective delivery of power by responding all the conditions and events occur at any stage such as generation, transmission, distribution, and consumption of electrical power with the adaptation of several strategies by using state of the art information communication technologies. For example, if any of the problems arise at the distribution side by the failure of medium voltage transformer, SG may have the capability to automatically recover and control the flow of electrical power at the distribution grid. Moreover, power demand profiles of the consumer may be shaped accordingly by smoothly adopting the demand profile of electrical power at the real-time and peak demand of electrical power in order to reduce the inclusive requirements of the power plant and its capital cost [4]. Figure 1 depicts the electrical grid system where power generated is transferred to distribution and control centers via high voltage transmission network which is further transferred to distribution feeders via medium voltage transmission network and at the very last stage is then transferred to meters via low voltage transmission network [5].

Although numerous conceptual prototypes and architectures are proposed for

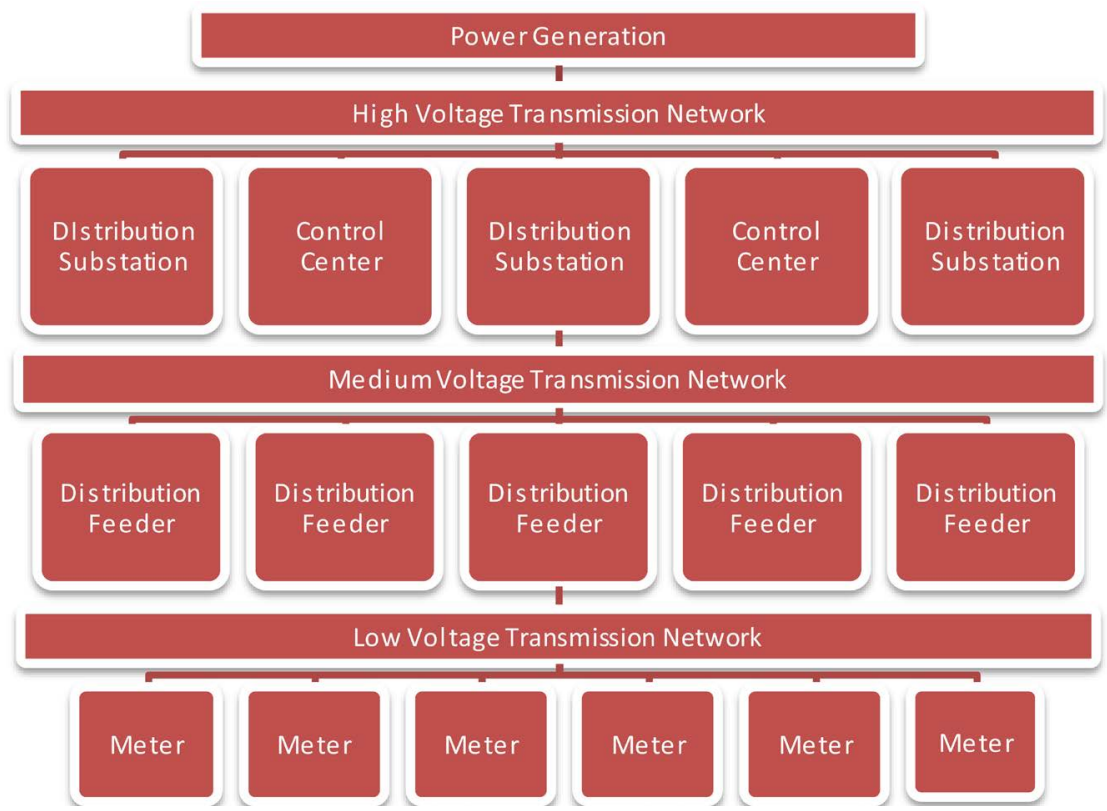

Figure 1. Electrical grid system outline [5]. 
the implementation of the SG. National Institute of Standards and Technology (NIST) proposes a conceptual architectural based model for SGs as a guideline for connecting, studying, analyzing and developing different SG standards. The overall organization of the paper is shown in Figure 2. The abstract level architecture to highlight different domains of SG is shown in Figure 3. These domains may have several subdomains and inter- and intra-domains communication requirements [6]. Requirements and Characteristics of Traditional and Smart Grid Infrastructures are highlighted in Table 1. Socio-Economic Challenges/Issues are depicted in Figure 4.

Smart grids are providing several novels applications such as Advanced Distribution Automation (ADA), Building/Home/Industrial Energy Management (BHIEM), Demand Response (DR), smart metering, Electrical Vehicles (EVs), etc. [8].

\section{Communication Networks of Smart Grid}

The infrastructure required for the environment of smart grid can be visualized

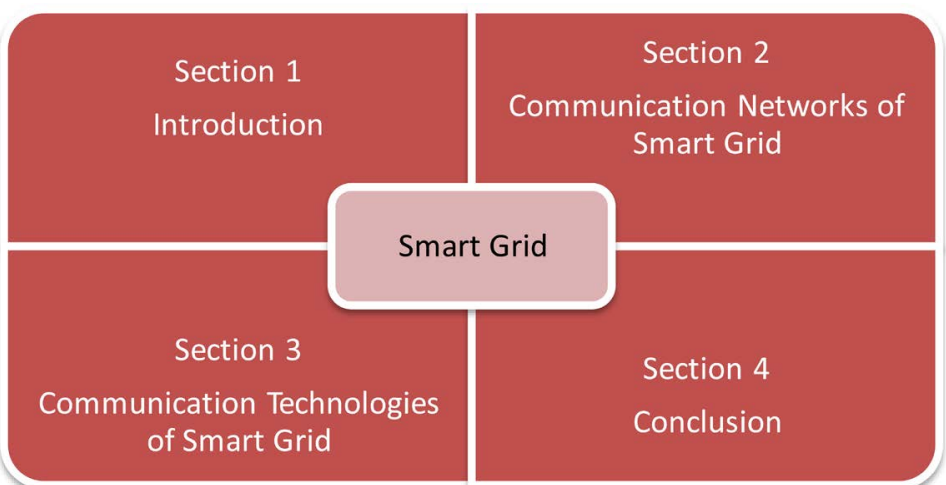

Figure 2. Organization of the paper.

Conceptual Model

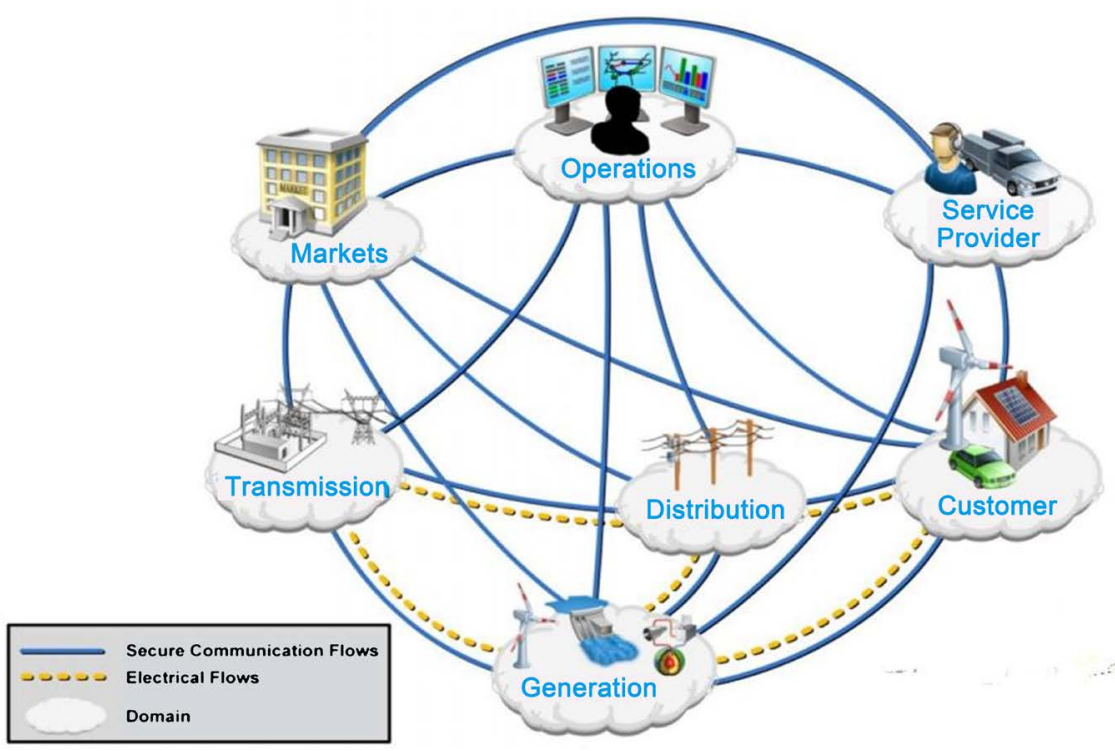

Figure 3. NIST conceptual model for Smart Grid [6]. 
as the hierarchical and layered architecture of all the major electrical and communication network elements of power generation to the distribution as shown in Figure 5.

Table 1. Comparison b/w traditional and smart grid [7].

\begin{tabular}{ccc}
\hline Parameter(s) & Traditional Grid & Smart Grid \\
\hline Flow of Information & Unidirectional & Bidirectional \\
Power Generation & Central & Distributed \\
Monitoring & Not Applicable & Self-Monitoring \\
Topology (Grid) & Radial & Network \\
Healing & Manual & Self \\
Testing & Manual & Remote \\
Control & Passive & Active \\
Efficiency (Overall) & Low & High \\
Environment-Friendly & No & Yes \\
\hline
\end{tabular}

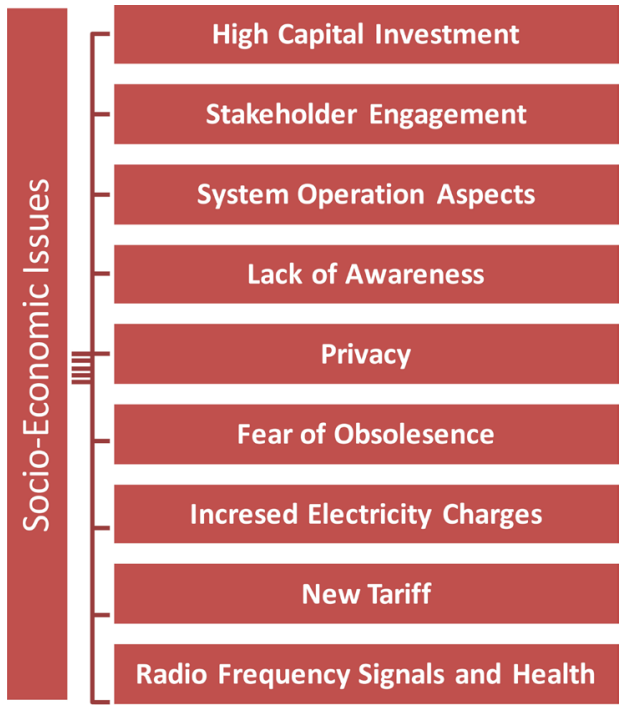

Figure 4. Socio-Economic challenges of SG [9].

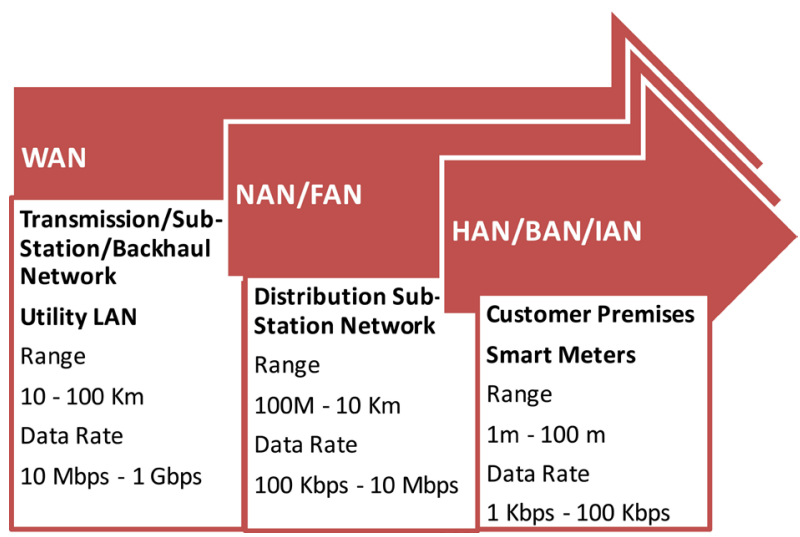

Figure 5. Communication Networks of Smart Grid (Requirements) [10] [13]. 
Three communication network architecture layers are based on wide area network (WAN), field area network (FAN)/neighbor area network (NAN) and home area network (HAN)/building area network (BAN)/industrial area network (IAN) [10] [11] [12]. HAN/BAN/IAN customer premises area CNAs provide home/building/industrial automation specific applications aim to send or receive the sensed data from electrical appliances to/from the controller embedded within the appliances. Main requirements are less power consumption, less cost, ease and secure links for communication. FAN/NAN based CNAs provide smart metering and distribution specific applications aim to send or receive the transmitted data from customer/field devices to/from the substation/concentrator. Main requirements are high data rate and large geographical coverage. WAN-based CNAs provide wide-area control, protection and monitoring aim to transmit a huge amount of data at a much higher data rate and longest distance [13].

\section{Communication Technologies of Smart Grid}

\subsection{Wireline Communication Technologies}

Wireline communication is always preferred due to the reliability and less prone to interference. All the communication technologies both in terms of wirleine and wireless are shown in Figure 6 and Figure 7. Modern technological trends such as Software Defined Networking (SDN), Internet of Things (IoT), New Radio (NR), and upcoming Fifth Generation (5 G) cellular networks based deployment of SGs are also highlighted (Table 2).

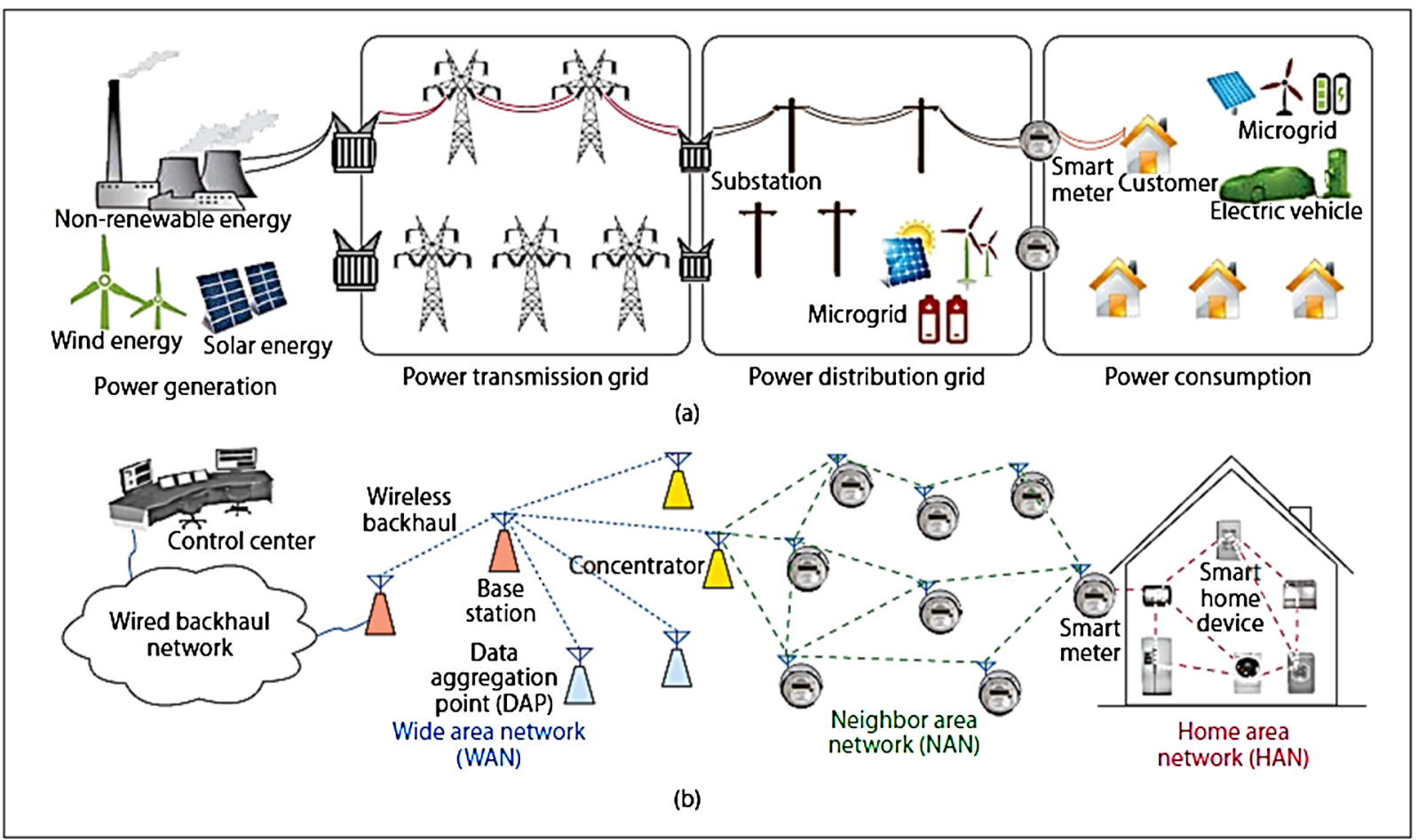

Figure 6. Complete Layered Architecture of SG [19]. 


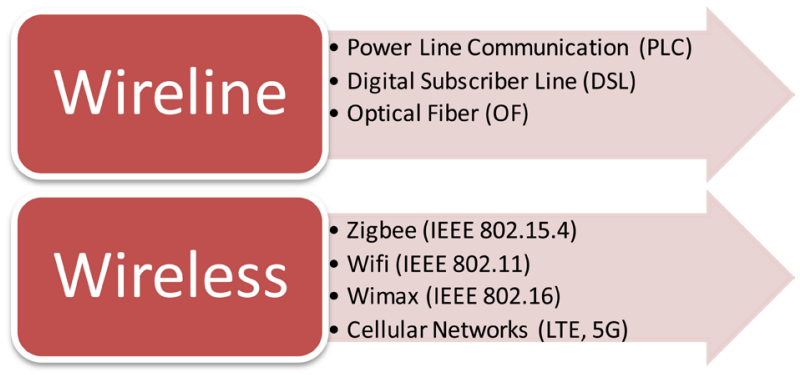

Figure 7. Communication Technologies of Smart Grid.

Table 2. Emerging Trends to enhance Modern SG Infrastructures.

\begin{tabular}{cc}
\hline Modern Trends and Technologies & Articles \\
Narrowband Internet of Things (IoT) based SG & {$[22]$} \\
NR based SG & {$[23][24]$} \\
SDN based SG & {$[25]$} \\
SDN and IoT based SG & {$[26]$} \\
SDN and Cloud-based SG & {$[27]$} \\
Renewable Energy Resources and SG & {$[28]$} \\
Distributed Power Control and SG & {$[29]$} \\
Energy Internet & {$[30]$} \\
\hline
\end{tabular}

\subsubsection{Digital Subscriber Line (DSL)}

Digital Subscriber Line (DSL) provides $10 \mathrm{Mbps}$ to $10 \mathrm{Gbps}$ data rate over the conventional telephone line. Asymmetric DSL (ADSL) provides 8 Mbps, ADSL2+ provides $24 \mathrm{Mbps}$ and very-high-bit-rate DSL (VDSL) provides $52 \mathrm{Mbps}$ downstream data rate over copper wires [14].

\subsubsection{Power Line Communication (PLC)}

Power Line Communication (PLC) is a widely used wireline communication technology for the SG. PLC face lots of technical challenges such as unpredicted propagation features and electromagnetic interference due to transformers and of transmission and distribution power lines. Cater to these issues, there are several PLC technologies are in use. Narrowband PLC (NB-PLC) provides 1 bps to $500 \mathrm{Kbps}$ data rate at $500 \mathrm{kHz}$ frequency whereas broadband PLC (BB-PLC) provides up to $200 \mathrm{Mbps}$ data rate at $2 \mathrm{MHz}$ to $30 \mathrm{MHz}$ frequency [14] [15]. NB-PLC and BB-PLC are based on two-way communication and are capable of handling and identifying equipment faults by delivering utility application specific high-speed real-time data. These technologies are preferable on the power grid distribution side by participating in and supporting distributed generation (DG), microgrids and consumer participation. PLCs are providing point to point (P2P) communication $\mathrm{b} / \mathrm{w}$ transformer substation on a medium voltage (MV) distribution and are configured to provide point to multipoint connectivity on a low voltage (LV) b/w meters and transformers near to the home, building and industry consumers [16] [17]. 


\subsubsection{Optical Fiber (OF)}

Optical fiber guided media communication is a globally deployed wireline communication infrastructure and is a great choice as a backbone network for SGs services like Video traffic with very low latency at very high speed. It provides a maximum of $10 \mathrm{Gbps}$ data rate with a single wavelength and $40 \mathrm{Gbps}$ to 1600 Gbps with wavelength division multiplexing (WDM). optical/electric transducers used in optical communication is an ideal choice for SG due to excellent sensing and measurement capabilities of the current and voltage values of electrical power [18].

\subsection{Wireless Communication Technologies}

Wireless communication technologies are always best suitable due to ease of implementation and less installation cost as a network to work with the smart grid. However wireless signals may have more attenuation and interference as that of wireline signals due to the direct impact of transmission and environmental factors, so these signals provide reliable communication over shorter distance with less data rate and bandwidth, also they always less secure and have serious privacy concerns [19].

\subsubsection{Zigbee (IEEE 802.15.4)}

ZigBee is IEEE 802.15.4 standard based on wireless mesh topology network for a cost-effective, low power and well-organized solution for wireless communications. ZigBee offers less data rate in personal area networks (PANs) such as HAN. This wireless technology provides numerous applications such as automation, control, messaging and remote monitoring of consumer electronics/home/building as well as healthcare, etc. It uses direct sequence spread spectrum (DSSS) to provide communication between linked devices in a very less power. It provides $250 \mathrm{kbps}$ data rate over the $2.4 \mathrm{GHz}$ unlicensed band, $40 \mathrm{kbps}$ over $915 \mathrm{MHz}$ band and $20 \mathrm{kbps}$ over $868 \mathrm{MHz}$ licensed band per channel. It supports 10 - 75 meters Point to point (P2P), 30 meters indoor and ever more in a mesh network. Mesh network may have multiple links to route data packets from source to destination and the links are dynamically updated and optimized by the network devices. These characteristics of the mesh network make it more scalable, stable and fault-tolerant network of wireless nodes [20].

\subsubsection{Wi-Fi (IEEE 802.11)}

Wireless Fidelity (Wi-Fi) is very popular and mature wireless local area network (WLAN) technology adopted by the home applications worldwide. It's operated in an unlicensed band and is subjected to interference because several other technologies are also sharing the same spectrum. Innovations in technologies are moving Wi-Fi towards power sketchy and reduced cost communication. It's very preferable technology for HAN architecture. However, citywide infrastructure of Wi-Fi will also support HAN, NAN and WAN applications. The typical data rate of Wi-Fi is $1-150$ Mbps over the distance of 20 to 100 meters. 


\subsubsection{WiMAX (IEEE 802.16)}

WiMAX can transport the application's data of terminal devices enabled with ZigBee or Wi-Fi wireless communication technologies in NAN and WAN networks. Smart meters (SMs) generated data is transferred from concentrators to the backend connected WiMAX base stations. It's a good choice for increased data to be transported via WiMAX base stations in a less cost making promising to deploy advanced real-time applications control with wider bandwidths. It also supports distributed automation, control, monitoring, management, fault identification oriented advanced SG applications. The typical data rate of WiMAX is 288.8 Mbps downlink and 72.2 Mbps uplink over the distance of 5 - 100 Kilometers.

\subsubsection{Cellular/Mobile Networks}

Cellular networks are most suitable wireless technology in WAN communication architecture for the transportations between SMs and the Utility companies due to its stable infrastructure. Cellular networks are offering numerous wider area services to the SG applications in a very affordable way. Emergent of third-generation $(3 \mathrm{G})$ and LTE wireless communication technologies to the cellular networks provide much higher data rates in NAN and WAN networks. Several grid assets such as circuit breakers (CBs), Sensors, transformers, remote terminal units (RTUs) and substations are connected to the nearly suitable centers via fiber connections, making it ideal for the SG applications to deploy in a short time frame without increasing the upfront cost of deployment. Typical data rate of Universal Mobile Telecommunications System (UMTS) example of $3 \mathrm{G}$ cellular is $2.048 \mathrm{Mbps}$ over the distance of up to 120 Kilometers and LTE is 300 Mbps downlink and 75 Mbps uplink over the distance of 100 Kilometers [21].

\section{Conclusion}

To efficiently implement the fully functional SG power systems for the management of real-time energy, numerous communication network architectures and technologies are essential to be deployed at each level of SG infrastructure from generation of electrical power to the distribution to substations, centers and then electrical feeders to the actual consumers of electricity, such as homes, buildings and industry. The division of the SG system is formed into three communications architectural networks, such as HAN, NAN, and WAN. For each network, several wired and wireless communication technologies are available and need to be critically evaluated before actual deployment, because each network may have different technological requirements in terms of data rate, coverage, frequency, reliability, security and cost. In this research article, concise review is presented in a systematic way by first introducing the general conventional infrastructure of the electrical power grid to more advanced and automated SG by introducing different network architectures and technologies for the communication, automation, and control. Several issues are present in SG infrastructure, communication network architectures and technologies for the deployment of SG, so it's a 
challenge to all the research community to devotedly work on possible solutions regarding SG real-world implementations.

\section{Conflicts of Interest}

The authors declare no conflicts of interest regarding the publication of this paper.

\section{References}

[1] Gungor, V.C., Lu, B. and Hancke, G.P. (2010) Opportunities and Challenges of Wireless Sensor Networks in Smart Grid. IEEE Transactions on Industrial Electronics, 57, 3557-3564. https://doi.org/10.1109/TIE.2009.2039455

[2] Wietfeld, C., et al. (2017) Smart Grids. IEEE Wireless Communications, 24, 8-9. https://doi.org/10.1109/MWC.2017.7909091

[3] Gungor, V., et al. (2012) Smart Grid and Smart Homes: Key Players and Pilot Projects. IEEE Industrial Electronics Magazine, 6, 18-34. https://doi.org/10.1109/MIE.2012.2207489

[4] Fang, X., Misra, S., Xue, G. and Yang, D. (2012) Smart Grid-The New and Improved Power Grid: A Survey. IEEE Communications Surveys \& Tutorials, 14, 944-980. https://doi.org/10.1109/SURV.2011.101911.00087

[5] Gao, J., Xiao, Y., Liu, J., Liang, W. and Chen, C.L.P. (2012) A Survey of Communication/Networking in Smart Grids. Future Generation Computer Systems, 28, 391-404. https://doi.org/10.1016/j.future.2011.04.014

[6] Usman, A. and Shami, S.H. (2013) Evolution of Communication Technologies for Smart Grid Applications. Renewable \& Sustainable Energy Reviews, 19, 191-199. https://doi.org/10.1016/j.rser.2012.11.002

[7] Alam, S., Sohail, M.F., Ghauri, S.A., Qureshi, I.M. and Aqdas, N. (2017) Cognitive Radio Based Smart Grid Communication Network. Renewable \& Sustainable Energy Reviews, 72, 535-548. https://doi.org/10.1016/j.rser.2017.01.086

[8] Dehalwar, V., Kalam, A., Kolhe, M.L. and Zayegh, A. (2016) Review of Machine to Machine Communication in Smart Grid. International Conference on Smart Grid and Clean Energy Technologies, ICSGCE, Chengdu, 19-22 October 2016, 134-139.

[9] Kappagantu, R. and Arul Daniel, S. (2018) Challenges \& Issues of Smart Grid Implementation: A Case of Indian Scenario. Journal of Electrical Systems and Information Technology, 5, 453-467.

[10] Kuzlu, M. and Pipattanasomporn, M. (2013) Assessment of Communication Technologies and Network Requirements for Different Smart Grid Applications. IEEE PES Innovative Smart Grid Technologies Conference (ISGT), Washington DC, 24-27 February 2013. https://doi.org/10.1109/ISGT.2013.6497873

[11] Raza, N., Jabbar, S., Han, J. and Han, K. (2018) Social Vehicle-to-Everything (V2X) Communication Model for Intelligent Transportation Systems Based on 5G Scenario. Proceedings of the 2 nd International Conference on Future Networks and Distributed Systems, Amman, 26-27 June 2018, Article No. 54. https://doi.org/10.1145/3231053.3231120

[12] Raza, N., Aftab, M.U., Akbar, M.Q., Ashraf, O. and Irfan, M. (2016) Mobile Ad-Hoc Networks Applications and its Challenges. Communications and Network, 8 , 131-136.

[13] Kuzlu, M., Pipattanasomporn, M. and Rahman, S. (2014) Communication Network 
Requirements for Major Smart Grid Applications in HAN, NAN and WAN. Computer Networks, 67, 74-88. https://doi.org/10.1016/j.comnet.2014.03.029

[14] Kabalci, Y. (2016) A Survey on Smart Metering and Smart Grid Communication. Renewable \& Sustainable Energy Reviews, 57, 302-318.

https://doi.org/10.1016/j.rser.2015.12.114

[15] Ercan, S.U., Ozgonenel, O. and Thomas, D.W.P. (2018) Power Line Communication Channel for Smart Grid. IEEE 6 th International Istanbul Smart Grids and Cities Congress and Fair (ICSG), Istanbul, 25-26 April 2018. https://doi.org/10.1109/SGCF.2018.8408974

[16] Galli, S., Scaglione, A. and Wang, Z. (2010) Power Line Communications and the Smart Grid. IEEE International Conference on Smart Grid Communications, Gaithersburg, 4-6 October 2010, 303-308. https://doi.org/10.1109/SMARTGRID.2010.5622060

[17] Yoon, S., Jang, S., Kim, Y. and Bahk, S. (2014) Opportunistic Routing for Smart Grid with Power Line Communication Access Networks. IEEE Transactions on Smart Grid, 5, 303-311. https://doi.org/10.1109/TSG.2013.2279184

[18] Khan, F., ur Rehman, A., Arif, M., Aftab, M. and Jadoon, B.K. (2016) A Survey of Communication Technologies for Smart Grid Connectivity. International Conference on Computing, Electronic and Electrical Engineering, Quetta, 11-12 April 2016, 256-261.

[19] Garau, M., Celli, G., Ghiani, E., Pilo, F. and Corti, S. (2017) Evaluation of Smart Grid Communication Technologies with a Co-Simulation Platform. IEEE Wireless Communications, 24, 42-49. https://doi.org/10.1109/MWC.2017.1600214

[20] Mahmood, A., Javaid, N. and Razzaq, S. (2015) A Review of Wireless Communications for Smart Grid. Renewable \& Sustainable Energy Reviews, 41, 248-260. https://doi.org/10.1016/j.rser.2014.08.036

[21] Quang-Dung, H., Yue, G. and Tho, L.-N. (2013) Challenges and Research Opportunities in Wireless Communication Networks for Smart Grid. IEEE Wireless Communications, 20, 89-95. https://doi.org/10.1109/MWC.2013.6549287

[22] Li, Y., Cheng, X., Cao, Y., Wang, D. and Yang, L. (2018) Smart Choice for the Smart Grid: Narrowband Internet of Things (NB-IoT). IEEE Internet of Things Journal, 5, 1505-1515. https://doi.org/10.1109/JIOT.2017.2781251

[23] Li, D., Sun, Z. and Zhao, X. (2018) Cognitive Radio-Based Smart Grids: From the Perspective of Full-Duplex Primary Users. IEEE Access, 6, 33088-33098. https://doi.org/10.1109/ACCESS.2018.2846764

[24] Alam, S., Malik, A.N., Qureshi, I.M., Ghauri, S.A. and Sarfraz, M. (2018) Clustering-Based Channel Allocation Scheme for Neighborhood Area Network in a Cognitive Radio Based Smart Grid Communication. IEEE Access, 6, 25773-25784. https://doi.org/10.1109/ACCESS.2018.2832246

[25] Rehmani, M.H., Davy, A., Jennings, B. and Assi, C. (2018) Software Defined Networks Based Smart Grid Communication: A Comprehensive Survey. arXiv:1801.04613.

[26] Chaudhary, R., et al. (2018) SDN-Enabled Multi-Attribute-Based Secure Communication for Smart Grid in IIoT Environment. IEEE Transactions on Industrial Informatics, 14, 2629-2640. https://doi.org/10.1109/TII.2018.2789442

[27] Abdeldjalil, C.D., Khoukhi, L. and Mouftah, H.T. (2018) Decentralized Cloud-SDN Architecture in Smart Grid: A Dynamic Pricing Model. IEEE Transactions on Industrial Informatics, 14, 1220-1231. https://doi.org/10.1109/TII.2017.2742147

[28] Rehmani, M.H., Reisslein, M., Rachedi, A., Erol-Kantarci, M. and Radenkovic, M. 
(2018) Integrating Renewable Energy Resources into the Smart Grid: Recent Developments in Information and Communication Technologies. IEEE Transactions on Industrial Informatics, 14, 2814-2825. https://doi.org/10.1109/TII.2018.2819169

[29] Yang, L., Liu, P., Li, L., Ma, K. and Liu, Z. (2018) Interference Compensation for Smart Grid Communications: A Distributed Power Control Approach. IEEE Access, 6, 18643-18654. https://doi.org/10.1109/ACCESS.2018.2820323

[30] Wang, K., Yu, J., Yu, Y., Qian, Y., Zeng, D., Guo, S., Xiang, Y. and Wu, J. (2018) A Survey on Energy Internet: Architecture, Approach, and Emerging Technologies. IEEE Systems Journal, 12, 2403-2416. https://doi.org/10.1109/JSYST.2016.2639820 